\title{
On the nature of errors in a choice reaction task'
}

HOWARD EGETH, THE JOHNS HOPKINS UNIVERSITY

EDWARD E. SMITH, BEHAVIORAL AND CLINICAL STUDIES RESEARCH CENTER, ST. ELIZABETHS HOSPITAL

The problem of dealing with incorrect responses in choice reaction time tasks is discussed. In the present experiment, incorrect responses tended to yield functions that were very similar to the functions plotted from correct responses. However, they averaged about $50 \mathrm{msec}$. faster than the correct responses. These observations were taken to mean that errors are generated by essentially the same processes as are correct responses, but that these processes are not taken to completion in the former case.

Whenever Ss serve in a choice reaction time task they will make some errors. Of course, the proportion of their responses that are incorrect is greatly influenced by such factors as stimulus discriminability, practice, S-R compatibility, and the relative emphasis placed on speed vs accuracy in their instructions. Nevertheless, it seems that at least occasional errors will occur even under the most favorable of circumstances. An important, but often unrecognized theoretical problem arises when an investigator decides how to handle his Ss' errors. Many strategies are open to an $E$, and he can generally find a precedent for any choice that he might make. For example, errors may be completely ignored (Shaffer, 1966), or they may be included with the correct RTs in the analysis (Morin \& Forrin, 1963). One specific procedure for including errors in the data anlysis is to use information transmission measures (Brainard, Irby, Fitts, \& Alluisi, 1962). Sometimes Ss are asked to correct their errors before proceeding to the next trial and only the total times for such corrected responses are included in the analysis (Williams, 1966). Finally, some investigators discuss the relative frequency of errors in various conditions without considering their times at all (Nickerson, 1966).

The purpose of the present paper is to describe the relation between correct and incorrect response latencies when they are subject to identical analyses. To this end, some data are examined that were originally collected for quite a different purpose.

\section{Method}

The S's task was one of character recognition. On each trial a letter of the alphabet was shown to him and he had to indicate whether or not it was a member of a predefined "target" set. There were two keys labeled "Yes" and "No" for this response. The independent variables were degree of practice (12 daily sessions) and size of target set (one, two, or four letters).
The Ss were three right-handed male college students, all with experience in other RT tasks. Their base rate of pay was $\$ 1.00$ per $h$ and they were awarded an additional $\$ .40$ whenever their mean RT in an experimental condition was faster than a predetermined criterion. For each error \$.05 was subtracted from their bonus. It was possible to earn a bonus of $\$ 1.20$ per session in addition to the $\$ 1.00$ base.

Stimuli were upper case pica typed letters. All 26 letters of the alphabet were used, and seven of them were chosen to serve as targets. Three sets-(K), (H and $S),(C, J, Q$, and $V$ )-were formed from these seven letters and they constituted the target sets used in the conditions where the number of targets were one, two, and four, respectively. In all conditions $53 \%$ of the stimulus presentations contained a target element. When the target set consisted of two or more elements all of them were used equally often and in a random order over trials. Also, all 19 non-target letters were used approximately equally often.

Each $S$ served in every condition during each of the 12 experimental sessions. The order of presentation of the three levels of number targets was balanced over three day periods. In each condition there were 42 trials, 10 practice followed by 32 test, for a total of 126 trials per session.

\section{Results and Discussion}

In Fig. 1 mean RT as a function of the size of the target set is plotted separately for correct and incorrect responses of both the "Yes" and "No"' types. Each curve represents data averaged over the three Ss for three days of practice. Both correct and incorrect responses show the following: generally increased speed with practice; greater slope between the one and two than between the two and four target conditions; and generally shorter times for "Yes" responses than "No" responses. The mean difference in RT between correct and incorrect responses was $50 \mathrm{msec}$.

The frequency of errors was unrelated to the size of the target set, but increased systematically from $9 \%$ on Days 1-3 to $22 \%$ on Days 10-12, with the overall average being $15 \%$.

In sum, incorrect responses seemed to follow the same pattern as correct responses since they were sensitive to the same task variables. The generality of this result is increased by Smith's (1965) finding that error RTs reflected the same relations as correct RTs with respect to number of targets, practice, 


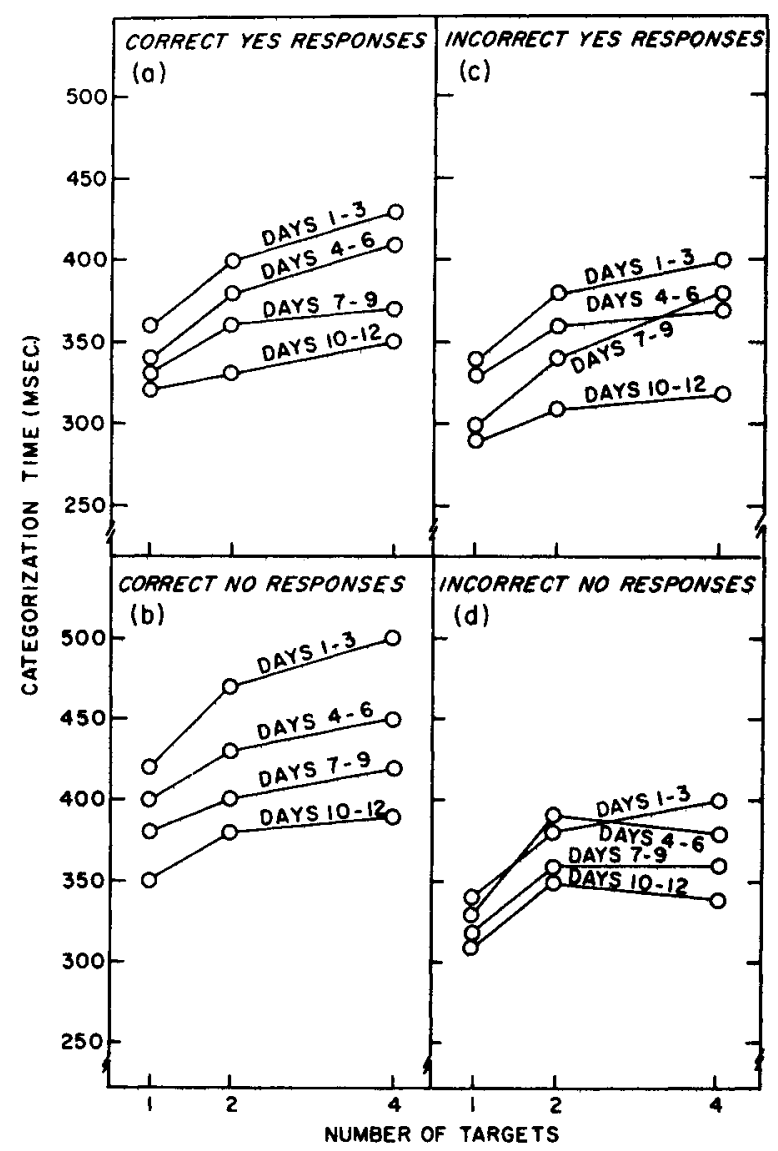

Fig. 1. Reaction times for correct "Yes" and "No" responses (panels a $\&$ b) and incorrect "Yes" and "No" responses (panels c \& d). Each point is an average for the three Ss. and type of response. In his experiment the targets were English words, and the target sets were defined either by category membership or enumeration. Such results seem to indicate that a conception of errors which makes them simply anticipations, a result of distraction, or some other kind of random response is untenable. The processes determining erroneous responses must be essentially the same as those determining correct responses. Since errors are faster, it also seems likely that the difference between correct and incorrect responses is that these processes are not carried to the same degree of completion on those trials that eventuate in errors.

\section{References}

Brainard, R. W., Irby, F. S., Fitts, P. M., \& Alluisi, E. A. Some variables influencing the rate of gain of information. $J$. exp. Psychol., 1962, 63, 105-110.

Morin, R. E., \& Forrin, B. Response equivocation and reaction time. J. exp. Psychol., 1963, 66, 30-36.

Nickerson, R. S. Response times with a memory-dependent decision task. J. exp. Psychol., 1966, 72, 761-769.

Shaffer, L.H. Some effects of partial advance information on choice reaction with fixed or variable S-R mapping. J. exp. Psychol., 1966, 72, 541-545.

Smith, E. E. The effects of familiarity on the perceptual recognition and categorization of verbal information. University of Michigan Technical Report, 05823-9-T, 1965.

Williams, J. A. Sequential effects in disjunctive reaction time: implications for decision models. J. exp. Psychol., 1966, 71, 665-672.

\section{Notes}

1. This research was supported by the Advanced Research Projects Agency, Department of Defense, and was monitored by the Air Force Office of Scientific Research, under contract No. AF 49(638)1235 with the Human Performance Center of the Department of Psychology, University of Michigan. 